\title{
GENERAL PARETO OPTIMAL ALLOCATIONS AND APPLICATIONS TO MULTI-PERIOD RISKS ${ }^{1}$
}

\author{
BY \\ Pauline BarRieu and Giacomo ScANDOlO 2
}

\begin{abstract}
In this paper, we consider the problem of Pareto optimal allocation in a general framework, involving preference functionals defined on a general real vector space. The optimization problem is equivalent to a modified sup-convolution of the different agents' preference functionals. The results are then applied to a multi-period setting and some further characterization of Pareto optimality for an allocation is obtained for expected utility for processes.
\end{abstract}

\section{KEYWORDS}

Pareto optimality, contract design, preference functional, multi-period risks.

\section{INTRODUCTION}

The question of Pareto optimal exchange of risk has been extensively studied in the insurance literature since the 1960s (see, for instance, the seminal papers by Borch (1960a, 1960b, 1960c and 1962), Bühlmann (1970, 1980 and 1984), Bühlmann and Jewell (1979), Gerber (1978), Raviv (1979), Wilson (1968)). This problem can indeed be easily related to the standard issue of optimal policy design in insurance: one agent is looking for an optimal "insurance" policy to cover her risk. In this sense, she can be seen as the "insured". On the other

\footnotetext{
1 Both authors would like to thank Ragnar Norberg for his very helpful comments and careful reading of previous versions of this work and two anonymous referees for their valuable comments and suggestions. Participants of the 31st SPA conference, Berlin Workshop on Climate Risk Securitization, ICMS Workshop on Credit Risk, XXX Amases conference, seminars at Cass Business School, ETH, Bocconi University, Louvain Workshop on New Actuarial Topics in Longevity and Transfer of Risks, Banff Workshop on Mathematics and the Environment, Sixth Scientific Conference on Insurance and Finance - DGVFM and Workshops on Quantitative Finance in Sydney, Venice and St Gallen are also thanked for stimulating discussions, and in particular, Knut Aase, Laura Ballotta, Marco Frittelli, Hans Foellmer, Dilip Madan, Marek Musiela, Fulvio Ortu and Fabio Trojani for their interesting suggestions.

2 Financial support by European Science Foundation under grant AMAMEF-963 is gratefully acknowledged.
} 
hand, another agent accepts to bear some risk, playing the role of "insurer" for the first agent. Finding the optimal transfer of risk between the two agents is equivalent to optimally design the insurance contract.

More recently, the question of optimal design of contracts has been raised with a financial perspective, when considering derivatives written on a nontradable risk or on a highly illiquid underlying asset (see for instance Barrieu and El Karoui (2005 and 2006), Jouini, Schachermayer and Touzi (2008), Acciaio (2007)). This issue has become even more essential as a consequence of both the recent development of insurance-linked securities (such as catbonds, weather derivatives, or, even more recently, bonds based upon a mortality or longevity index), and the greater sophistication of structured deals, as in credit risk securitization or in bespoke transactions between a bank and some hedge funds. Pricing is not the only issue any longer. The design of the transaction could ensure its feasibility. However, transferring risk in insurance and in finance is somehow different: risk in finance is taken in a wider sense as it represents the uncertain outcome associated with a given financial position. The sign of the realization does not a priori matter in the risk transfer design. The derivative market can be seen as a good illustration of this aspect: indeed, forward contracts, options or swaps have particular payoffs which are not directly related to any particular loss of the contract's seller.

The literature on Pareto optimal allocations of risk has focused so far on specific preference functionals: the classical framework adopted in the insurance literature (see for instance the seminal works by Borch (1962), Du Mouchel (1968) or Gerber (1978) among many others) is that of expected utility; more recently, the framework has been extended to convex risk measures (see for instance Barrieu and El Karoui (2005 and 2006) or Jouini, Schachermayer and Touzi (2008)). However, these two preference functionals are defined on some space of random variables. In this paper, a more general result on the characterization of Pareto optimal allocations is obtained for preference functionals defined on a general vector space. This extension of classical results to a general setting may have several possible applications. In particular, in this paper, we extend the notion of Pareto-optimality to a multi-period setting, involving risk streams taking place at different dates, and therefore potentially leading to risk exchanges at different times. Considering a multi-period setting is particularly relevant when looking at the design of some financial and/or insurance contracts based upon long-term risks such as longevity risk or climate risk. A potential application of this study lies therefore in the characterization of optimal features for the securitization process of such risks. The importance of this type of issues has been recently confirmed by the failure of the longevity bond issued by the European Investment Bank and BNP Paribas.

The rest of the paper is organized as follows. In Section 2, we briefly recall the main results on Pareto optimality in a one-period setting. Then, in Section 3, the key notions related to Pareto optimality are extended to a general framework, involving preference functionals defined on a general vector space and the characterization of optimal exchanges between two agents is obtained as 
a solution of a sup-convolution problem between their modified preference functionals. Section 4 is dedicated to the multi-period setting, where general necessary and sufficient conditions for Pareto optimality of a risk exchange are obtained. In Section 5, the results are illustrated in different relevant examples, where explicit characterizations of the transfer rule can be obtained. Finally, some concluding comments are presented in the last section.

\section{Pareto optimality in the ONE-Period CASE}

In this section we review some well-known facts about Pareto optimal allocations between two agents in a one-period setting, i.e. when the exchange of risk takes place at only one fixed time in the future. In particular, after defining the notion of Pareto optimality for general preference functionals defined on some subspaces of random variables, we characterize Pareto optimal allocations and determine the exchange rule in the expected utility framework.

\subsection{Pareto optimal allocations}

Let $T>0$ be a fixed maturity, $(\Omega, \mathcal{F}, \mathbb{P})$ be a standard probability space, where $\mathcal{F}$ is the (common) information available to the different agents at time $T$. Moreover, let $L^{0}$ (resp. $L^{1}$ and $L^{\infty}$ ) be the space of all random variables (resp. integrable and bounded random variables) defined on $(\Omega, \mathcal{F}, \mathbb{P})$. Let $L \subset L^{0}$ be a subspace collecting all risks of interest, where a risk is an uncertain future monetary payoff. We convene that a positive value of $X$ means an income, while a negative value means a loss. Finally, the expectation $\mathbb{E}_{\mathbb{P}}$ will be simply denoted by $\mathbb{E}$ and every (in)equality involving random variables will be understood $\mathbb{P}$-a.s.

In this framework, there are two agents, $\mathrm{A}$ and $\mathrm{B}$, who want to partially exchange their risks. The agents may simply be the two parties in a reinsurance contract or in a OTC derivative contract. Therefore, it may not be always clear which party acts as the seller or the buyer.

The agents' preferences over future risks are described by two preference functionals $U_{A}$ and $U_{B}$. Here and in what follows, a preference functional is a real valued functional $U$ defined on a suitable subspace of random variables $L$ and such that

$$
X \succeq_{i} Y \Longleftrightarrow U_{i}(X) \geq U_{i}(Y),
$$

where $\succeq_{i}$ denotes the preference structure of the agent $i(i=A, B)$ over risks in $L$. A typical example of such preference functionals is the standard expected utility, but also any monetary utility functional, the opposite of a convex risk measure, or that of a cash sub-additive risk measure as introduced by El Karoui and Ravanelli (2007). Some further assumptions on these functionals will be made later.

In our framework, both agents initially face some individual risks described by the random variables $\widehat{X}^{A}, \widehat{X}^{B} \in L$, respectively; we denote the aggregated 
risk by $\widehat{X}=\widehat{X}^{A}+\widehat{X}^{B}$. They may design and enter a contract, through which they may partially exchange some of their risks, in order to achieve better positions. It is however clear that the two optimization problems usually conflict each other, since the aggregate risk must remain the same.

Definition 2.1 Let $\widehat{X} \in L$ be an initial aggregate risk. An allocation of $\widehat{X}$ between agent $\mathrm{A}$ and agent $\mathrm{B}$ is a pair of risks $\left(X^{A}, X^{B}\right) \in L \times L$ satisfying

$$
X^{A}+X^{B}=\widehat{X} \text {. }
$$

The set of all possible allocations of $\widehat{X}$ will be denoted by $\mathcal{A}(\widehat{X})$ and the pair $\left(\widehat{X}^{A}, \widehat{X}^{B}\right) \in \mathcal{A}(\widehat{X})$ will be called the initial allocation.

The following dominance relation combines the preferences of both agents.

Definition 2.2 Given two allocations $\left(X^{A}, X^{B}\right)$ and $\left(Y^{A}, Y^{B}\right)$ we say that the former dominates the latter if

$$
U_{A}\left(X^{A}\right) \geq U_{A}\left(Y^{A}\right) \text { and } U_{B}\left(X^{B}\right) \geq U_{B}\left(Y^{B}\right),
$$

i.e. if both agents weakly prefer the former allocation. The dominance is strict if at least one of the two inequalities is strict.

It is then possible to introduce an optimality criterion for an allocation, in the following classical sense:

Definition 2.3 An allocation $\left(X^{A}, X^{B}\right) \in \mathcal{A}(\widehat{X})$ is Pareto optimal if it is not strictly dominated by another allocation in $\mathcal{A}(\widehat{X})$.

Explicitly, an allocation $\left(X^{A}, X^{B}\right)$ is Pareto optimal if for any $\left(Y^{A}, Y^{B}\right) \in \mathcal{A}(\widehat{X})$ the following two implications hold true:

$$
\begin{aligned}
& U_{A}\left(Y^{A}\right)>U_{A}\left(X^{A}\right) \Longrightarrow U_{B}\left(Y^{B}\right)<U_{B}\left(X^{B}\right), \\
& U_{B}\left(Y^{B}\right)>U_{B}\left(X^{B}\right) \Longrightarrow U_{A}\left(Y^{A}\right)<U_{A}\left(X^{A}\right) .
\end{aligned}
$$

Plainly, an agent will accept a risk allocation if this improves his initial situation, that is if the new risk allocation dominates the initial one. Such a condition is usually referred to as the rationality condition. Therefore, in addition to the Pareto optimality condition, an optimal risk allocation should also satisfy individual rationality conditions, ensuring the participation of the different agents. However, Pareto-optimal allocations need not satisfy this rationality condition. In this paper, in order to preserve the clarity of our results, we focus on the Pareto optimality condition only and therefore we do not address the issue of rationality.

As Pareto optimality is a natural necessary condition for a risk exchange to take place, a large body of literature has been devoted to obtain alternative 
characterizations and to state existence results for Pareto optimal allocations. This paper is a contribution to the first problem, by considering this issue in a (much) more general setting than the standard one we recall below.

A classical characterization of Pareto optimality is possible under the two following assumptions. First $U_{A}$ and $U_{B}$ do not attain a maximum on $L$ : this assumption, referred to as the Non-Satiation Property $(\mathcal{N} S)$, is rather natural as it means that there is no "best" risk for the two agents, or in economic terms, that the two agents are never satiated. Second, both $U_{A}$ and $U_{B}$ are supposed to be concave, i.e.

$$
U(\alpha X+(1-\alpha) Y) \geq \alpha U(X)+(1-\alpha) U(Y) \forall X, Y \in L, \alpha \in[0,1],
$$

translating the idea of risk aversion.

In this case, the notion of Pareto optimality is closely related to the supconvolution of $U_{A}$ and $\lambda U_{B}$, for a given constant $\lambda>0$, i.e. to the following optimization problem

$$
\left.\left[U_{A} \square \lambda U_{B}\right] \widehat{X}\right) \triangleq \sup _{\left(Y^{A}, Y^{B}\right) \in \mathcal{A}(\widehat{X})}\left\{U_{A}\left(Y^{A}\right)+\lambda U_{B}\left(Y^{B}\right)\right\} .
$$

Plainly, we say that an allocation solves ( $\lambda$-SC) if the above supremum is attained. Note that this optimization program involves a representative agent of the market with the preference functional $U_{\widehat{X}}(Y)=U_{A}(Y)+\lambda U_{B}(\widehat{X}-Y)$. More precisely,

Proposition 2.4 Under the above assumptions for $U_{A}$ and $U_{B}$, an allocation is Pareto optimal if and only if it solves $(\lambda-S C)$ for some $\lambda>0$.

Proof. Gerber (1978) first proved this equivalence. This is however an immediate corollary of the more general Theorem 3.2 to be proved below: therefore, we omit its proof.

\subsection{The case of expected utilities}

We now assume that the preference functionals of both agents can be written in the form of expected utilities, i.e.

$$
U_{A}(X)=\mathbb{E} u_{A}(X) \quad \text { and } \quad U_{B}(X)=\mathbb{E} u_{B}(X),
$$

where $u_{A}$ and $u_{B}$ are two utility functions, in the following sense:

Definition 2.5 A utility function is a differentiable map $u: \mathbb{R} \rightarrow \mathbb{R}$ that is concave and strictly increasing.

A typical example of utility function is provided by the exponential utility: $u(x)=-\exp (-\gamma x)$ for a fixed risk aversion coefficient $\gamma>0$. 
The domain of definition of $U_{A}$ and $U_{B}$ strongly depends on the utility functions $u_{A}$ and $u_{B}$ and in general it is not a subspace. However, it surely contains $L^{\infty}$. Therefore we do not attempt to give a precise definition of $L$, but we assume throughout this section that $L^{\infty} \subseteq L$.

Note that it is possible to give a broader definition, where a utility function is defined on a half-line like $(0,+\infty)$; this wider class includes for instance the logarithmic-type utilities. However, for the sake of clarity in the discussion, we focus on utility functions defined on all the real line. The attitude towards risk of any agent having a twice differentiable utility function $u$ can be further described by the Arrow-Pratt coefficient of absolute risk aversion, as follows:

Definition 2.6 Let $u$ be a twice differentiable utility function. The Arrow-Pratt coefficient of absolute risk aversion is defined as:

$$
R A(x)=-\frac{u^{\prime \prime}(x)}{u^{\prime}(x)}, \quad x \in \mathbb{R} .
$$

This coefficient characterizes how the risk aversion of the agent changes as his wealth increases. For the exponential utility function $R A(x) \equiv \gamma$ is constant.

Finally, the following basic fact will be useful when characterizing Pareto optimal allocations:

Lemma 2.7 If $u$ is a utility function, $S \in \mathcal{F}$ and $X \in L$ is such that $I_{S} u^{\prime}(X-\varepsilon) \in$ $L^{1}$ for some $\varepsilon>0$, then the map $h(\alpha) \triangleq \mathbb{E} u\left(X+\alpha I_{S}\right)$ is differentiable at $\alpha=0$ and:

$$
h^{\prime}(0)=\mathbb{E}\left[I_{S} u^{\prime}(X)\right]
$$

Proof. As $u$ is concave, we know that, for any $x, y \in \mathbb{R}$ :

$$
|u(x+y)-u(x)|=u(x+y)-u(x) \leq y u^{\prime}(x)=|y| u^{\prime}(x) \text { when } y>0 .
$$

and

$|u(x+y)-u(x)|=u(x)-u(x+y) \leq-y u^{\prime}(x+y)=|y| u^{\prime}(x+y)$ when $y<0$.

Since $u^{\prime}>0$ it follows that for any $y \in \mathbb{R}$ :

$$
|u(x+y)-u(x)| \leq|y| \max \left\{u^{\prime}(x), u^{\prime}(x+y)\right\} .
$$

Consequently, for any $\alpha \neq 0$ :

$$
\begin{aligned}
\left|\frac{u\left(X+\alpha I_{S}\right)-u(X)}{\alpha}\right| & \leq\left|I_{S}\right| \max \left\{u^{\prime}(X), u^{\prime}\left(X+\alpha I_{S}\right)\right\} \\
& =\max \left\{I_{S} u^{\prime}(X), I_{S} u^{\prime}\left(X+\alpha I_{S}\right)\right\} \in L^{1}
\end{aligned}
$$


Indeed since $X \geq X-\varepsilon$ and, for $|\alpha| \leq \varepsilon$ :

$$
X+\alpha I_{S} \geq X-\varepsilon,
$$

we have $0 \leq I_{S} u^{\prime}(X), I_{S} u^{\prime}\left(X+\alpha I_{S}\right) \leq I_{S} u^{\prime}(X-\varepsilon) \in L^{1}\left(u^{\prime}\right.$ decreasing) and then both $I_{S} u^{\prime}(X), I_{S} u^{\prime}\left(X+\alpha I_{S}\right)$ are in $L^{1}$ (and so is their max).

On the other hand, we have

$$
\frac{u\left(X+\alpha I_{S}\right)-u(X)}{\alpha} \rightarrow I_{S} u^{\prime}(X) \text { as } \alpha \rightarrow 0 .
$$

Then, we conclude, using the bounded convergence theorem,

$h^{\prime}(0)=\lim _{\alpha \rightarrow 0} \frac{\mathbb{E} u\left(X+\alpha I_{S}\right)-\mathbb{E} u(X)}{\alpha}=\mathbb{E} \lim _{\alpha \rightarrow 0} \frac{u\left(X+\alpha I_{S}\right)-u(X)}{\alpha}=\mathbb{E}\left[I_{S} u^{\prime}(X)\right]$.

\subsubsection{A characterization of Pareto optimality}

In view of the concavity and strict monotonicity of $u$, the functional $U(\cdot)=\mathbb{E} u(\cdot)$ satisfies the assumptions of Proposition 2.4. In particular, $U$ satisfies the NonSatiation Property $(\mathcal{N} S)$, as it cannot attain a maximum on $L$ : for any $X \in L$, $\mathbb{E} u(X+1)>\mathbb{E} u(X)$. In this setting, a further and more explicit characterization of Pareto optimality can be obtained:

Theorem 2.8 Let $\left(X^{A}, X^{B}\right) \in \mathcal{A}(\widehat{X})$; then the following statements are equivalent:

1. $\left(X^{A}, X^{B}\right)$ is Pareto optimal;

2. $\left(X^{A}, X^{B}\right)$ solves $(\lambda-S C)$ for some $\lambda>0$;

3. The equality

$$
u_{A}^{\prime}\left(X^{A}\right)=\lambda u_{B}^{\prime}\left(X^{B}\right)
$$

holds for some constant $\lambda>0$ (the same as at point 2 ).

Remark 2.9 The equivalence $1 \Leftrightarrow 3$ was heuristically proved by Borch (1962) and then more rigorously by Du Mouchel (1968). However, even this latter proof required implicitly some integrability conditions for $u_{A}^{\prime}\left(X^{A}\right)$ and $u_{B}^{\prime}\left(X^{B}\right)$ that we do not assume here.

Proof. 1. $\Leftrightarrow 2$. See Proposition 2.4 or, more generally, Theorem 3.2.

2. $\Rightarrow 3$. Let $\left(X^{A}, X^{B}\right)$ be an allocation solving $(\lambda$-SC) for some $\lambda>0$ and set for simplicity

$$
W_{A} \triangleq u_{A}^{\prime}\left(X^{A}\right) \quad \text { and } \quad W_{B} \triangleq u_{B}^{\prime}\left(X^{B}\right) .
$$


Note that $W_{A}, W_{B}>0$ as both $u_{A}$ and $u_{B}$ are strictly increasing. For any $k \in \mathbb{N}$, any bounded interval $C \subset \mathbb{R}$ and any $\alpha \in \mathbb{R}$ we can build the following admissible allocation

$$
\left\{\begin{array}{l}
Y^{A}(\alpha, k, C) \equiv X^{A}+\alpha I_{S(k, C)} \\
Y^{B}(\alpha, k, C) \equiv X^{B}-\alpha I_{S(k, C)}
\end{array}\right.
$$

where

$$
S(k, C) \triangleq\left\{W_{A}, W_{B} \leq k, W_{A}-\lambda W_{B} \in C\right\} \in \mathcal{F} .
$$

Since $\left(X^{A}, X^{B}\right)$ solves $(\lambda-\mathrm{SC})$, the map

$$
f(\alpha) \triangleq \mathbb{E} u_{A}\left(Y^{A}(\alpha, k, C)\right)+\lambda \mathbb{E} u_{B}\left(Y^{B}(\alpha, k, C)\right), \alpha \in \mathbb{R} .
$$

clearly attains a maximum at $\alpha=0$, for any fixed $k$ and $C$. Moreover, by definition of $S(k, C)$, we have that $I_{S(k, C)} u^{\prime}\left(X^{A}\right) \in L^{\infty}$; using continuity of $u^{\prime}$, it is immediate to check that for some (actually, for any) $\varepsilon>0$ it holds $I_{S(k, C)}$ $u^{\prime}\left(X^{A}-\varepsilon\right) \in L^{\infty}$ and thus Lemma 2.7 can be applied obtaining:

$$
f_{A}^{\prime}(0)=\mathbb{E}\left[I_{S(k, C)} W_{A}\right],
$$

where $f_{A}(\alpha) \triangleq \mathbb{E} u_{A}\left(Y^{A}(\alpha, k, C)\right)$. In a similar way we prove that $f_{B}^{\prime}(0)=$ $\mathbb{E}\left[I_{S(k, C)} W_{B}\right]$, where $f_{B}(\alpha) \triangleq \mathbb{E} u_{B}\left(Y^{B}(\alpha, k, C)\right)$. As a consequence, the map $f$ is differentiable at $\alpha=0$ and $f^{\prime}(0)=\mathbb{E}\left[I_{S(k, C)}\left(W_{A}-\lambda W_{B}\right)\right]$. The first order condition for a maximum is then:

$$
\mathbb{E}\left[I_{S(k, C)}\left(W_{A}-\lambda W_{B}\right)\right]=0 \quad \forall k, C .
$$

For any fixed $C$, we have:

$$
I_{S(k, C)}\left(W_{A}-\lambda W_{B}\right) \rightarrow I_{\left\{W_{A}-\lambda W_{B} \in C\right\}}\left(W_{A}-\lambda W_{B}\right) \quad \mathbb{P} \text {-a.s. as } k \rightarrow \infty .
$$

Moreover,

$$
\left|I_{S(k, C)}\left(W_{A}-\lambda W_{B}\right)\right| \leq 1_{\left\{W_{A}-\lambda W_{B} \in C\right\}}\left|W_{A}-\lambda W_{B}\right| \in L^{1} .
$$

Therefore, by using the bounded convergence theorem, we get

$$
\mathbb{E}\left[I_{\left\{W_{A}-\lambda W_{B} \in C\right\}}\left(W_{A}-\lambda W_{B}\right)\right]=\lim _{k \rightarrow \infty} \mathbb{E}\left[I_{S(k, C)}\left(W_{A}-\lambda W_{B}\right)\right]=0 .
$$

As $C$ is a generic bounded interval, we can conclude that $W_{A}-\lambda W_{B}$ vanishes $\mathbb{P}$-a.s. and we obtain the desired result (statement 3). 
3. $\Rightarrow 2$. Let us now prove that if $u_{A}^{\prime}\left(X^{A}\right)=\lambda u_{B}^{\prime}\left(X^{B}\right)$, then $\left(X^{A}, X^{B}\right)$ solves $(\lambda$-SC). If $\left(Y^{A}, Y^{B}\right)$ is another allocation, then, by concavity of $u_{A}$ and $u_{B}$ we have:

$$
\begin{aligned}
& u_{A}\left(Y^{A}\right) \leq u_{A}\left(X^{A}\right)+u_{A}^{\prime}\left(X^{A}\right)\left(Y^{A}-X^{A}\right), \\
& u_{B}\left(Y^{B}\right) \leq u_{B}\left(X^{B}\right)+u_{B}^{\prime}\left(X^{B}\right)\left(Y^{B}-X^{B}\right) .
\end{aligned}
$$

Multiplying the second inequality by $\lambda>0$ and adding it to the first one, we obtain:

$$
u_{A}\left(Y^{A}\right)+\lambda u_{B}\left(Y^{B}\right) \leq u_{A}\left(X^{A}\right)+\lambda u_{B}\left(X^{B}\right)+\theta,
$$

where $\theta=u_{A}^{\prime}\left(X^{A}\right)\left(Y^{A}-X^{A}\right)+\lambda u_{B}^{\prime}\left(X^{B}\right)\left(Y^{B}-X^{B}\right)=u_{A}^{\prime}\left(X^{A}\right)\left(Y^{A}+Y^{B}-X^{A}-X^{B}\right)=0$. Therefore $\left(X^{A}, X^{B}\right)$ solves $(\lambda$-SC).

Remark 2.10 If both $u_{A}$ and $u_{B}$ are linear utilities (in other words, when both agents are risk-neutral), then any allocation $\left(X^{A}, X^{B}\right) \in \mathcal{A}(\widehat{X})$ is Pareto optimal.

Remark 2.11 The third statement of the theorem involves the following equality of the marginal utilities of both agents $\frac{u_{A}^{\prime}\left(X^{A}\right)}{u_{B}^{\prime}\left(X^{B}\right)}=\lambda$. Using the relationship between marginal utility and state price density (see for instance Duffie (1996)), we can reinterpret this condition as both agents agreeing on their pricing measure. This seems very intuitive: the beliefs of both agents on the distribution of risks should coincide for the Pareto-optimal allocation.

\subsubsection{Characterization of Pareto optimal allocation rules}

After the general characterization of Pareto optimal allocations, we are now interested in finding some rules on how to obtain these allocations. We need first to establish what an appropriate rule should be.

Formally, the considered $\sigma$-algebra $\mathcal{F}$ collects all the information available to both agents at time $T$ and the risk allocation structure must be based upon this information. It is however often the case that the two agents seek for allocations based only on the observation of the "realized" risks $\left(\widehat{X}^{A}, \widehat{X}^{B}\right)$. This corresponds to setting $\mathcal{F}=\sigma\left(\widehat{X}^{A}, \widehat{X}^{B}\right)=\sigma\left(\widehat{X}, \widehat{X}^{A}\right)$ and leads to new risk allocations of the form

$$
\left\{\begin{array}{l}
X^{A}=\phi\left(\widehat{X}, \widehat{X}^{A}\right), \\
X^{B}=\widehat{X}-X^{A},
\end{array}\right.
$$

for some measurable function $\phi: \mathbb{R}^{2} \rightarrow \mathbb{R}$. Note that $\mathcal{F}$ is also $\sigma\left(\widehat{X}, \widehat{X}^{B}\right)$ and therefore both agents A and B play a perfectly symmetric role. More formally,

Definition 2.12 An allocation rule is a real valued measurable map $\phi=\phi\left(x, x^{A}\right)$ of two real arguments. For any initial risks $\widehat{X}$ (aggregated) and $\widehat{X}^{A}$ (for agent A), the associated allocation is defined in (2). 
If $\phi$ does not depend on $x^{A}$, then the associated allocation rule is called a pool rearrangement or, particularly in the actuarial literature, a mutual reinsurance treaty.

Definition 2.13 An allocation rule $\phi$ is said to be Pareto optimal if, for any choice of $\widehat{X}$ and $\widehat{X}^{A}$, the associated allocation is Pareto optimal.

In the expected utility framework, it turns out that Pareto optimal allocations come always in the form of pool rearrangements, and therefore do not depend on the individual risk exposure of the different agents. Moreover, if $u^{\prime \prime}$ exists, such allocations can be expressed in terms of the Arrow-Pratt coefficients of absolute risk aversion of the agents, previously introduced in Definition 2.6 and denoted by $R A_{i}$ for $i=A, B$. The following proposition is due to Wyler (1990), who extended a result of Borch (1960a) and Bühlmann (1984):

Proposition 2.14 If $u_{A}$ and $u_{B}$ are strictly concave utility functions, then the following are equivalent:

1. $\left(X^{A}, X^{B}\right)$ is Pareto optimal;

2. $X^{A}=\phi(\widehat{X})$ where the map $\phi$ satisfies for some constant $\lambda>0$ :

$$
u_{A}^{\prime}(\phi(\widehat{X}))=\lambda u_{B}^{\prime}(\widehat{X}-\phi(\widehat{X})) ;
$$

3. Provided $u_{A}$ and $u_{B}$ are twice differentiable: $X^{A}=\phi(\widehat{X})$ where the map $\phi$ is differentiable and solves for any $x \in \mathbb{R}$ :

$$
\phi^{\prime}(x)=\frac{R A_{B}(x-\phi(x))}{R A_{A}(\phi(x))+R A_{B}(x-\phi(x))} .
$$

Remark 2.15 In principle the allocation rule $\phi$ may depend on the distribution of $\left(\widehat{X}, \widehat{X}^{A}\right)$. However, in the case of expected utilities, the above result shows that the allocation rule is independent from the distribution of the risks.

Remark 2.16 Wyler (1990) also proved an existence and uniqueness result for the equation (4) under the boundary condition $\phi(0)=$ constant. Thus, also equation (3) will have a unique solution provided $\lambda=\frac{u_{A}^{\prime}(\phi(0))}{u_{B}^{\prime}(-\phi(0))}$.

\section{A general result on Pareto optimality on a ReAl Vector space}

In this section, we study the problem of Pareto optimality in a rather abstract setting. The purpose of this section is to present a general mathematical result, which will be especially useful in the next section where we will consider spaces of processes in a multi-period framework. The interpretation of the abstract result will be done later when considering potential applications. More precisely, we now consider a general real vector space $\mathcal{L}$ and obtain a characterization 
result of "Pareto optimal allocations" as solutions of a sup-convolution problem, extending the equivalence between statements 1 . and 2 . of Theorem 2.8 and Proposition 2.4.

\subsection{Framework and definitions}

Let $\mathcal{L}$ be a real vector space whose elements $x \in \mathcal{L}$ have to be interpreted in this section as some sort of "risks". We can formally generalize the concepts introduced in Section 2. In particular, $U_{A}, U_{B}: \mathcal{L} \rightarrow \mathbb{R}$ are the preference functionals of the agents in the sense that agent $\mathrm{A}$ (resp. agent $\mathrm{B}$ ) weakly prefers $x$ over $y(x, y \in \mathcal{L})$ if and only if $U_{A}(x) \geq U_{A}(y)$ (resp. $U_{B}(x) \geq U_{B}(y)$ ). Moreover,

Definition 3.1 1. Given an initial risk $\hat{x} \in \mathcal{L}$, an allocation is a couple $\left(x^{A}, x^{B}\right) \in$ $\mathcal{L} \times \mathcal{L}$ such that $x^{A}+x^{B}=\hat{x}$. The set $\mathcal{A}(\hat{x})$ collects all allocations.

2. An allocation $\left(x^{A}, x^{B}\right) \in \mathcal{A}(\hat{x})$ is said to be Pareto optimal if the following two implications hold for any other allocation $\left(y^{A}, y^{B}\right) \in \mathcal{A}(\hat{x})$ :

$$
\begin{aligned}
& U_{A}\left(y^{A}\right)>U_{A}\left(x^{A}\right) \Longrightarrow U_{B}\left(y^{B}\right)<U_{B}\left(x^{B}\right), \\
& U_{B}\left(y^{B}\right)>U_{B}\left(x^{B}\right) \Longrightarrow U_{A}\left(y^{A}\right)<U_{A}\left(x^{A}\right)
\end{aligned}
$$

3. An allocation $\left(x^{A}, x^{B}\right) \in \mathcal{A}(\hat{x})$ is said to solve the $\lambda$-Sup Convolution problem $(\lambda$-SC) for a given $\lambda>0$ if:

$$
U_{A}\left(x^{A}\right)+\lambda U_{B}\left(x^{B}\right)=\left[U_{A} \square_{\lambda} U_{B}\right](\hat{x}) \triangleq \sup _{\left(y^{A}, y^{B}\right) \in \mathcal{A}(\hat{x})}\left\{U_{A}\left(y^{A}\right)+\lambda U_{B}\left(y^{B}\right)\right\}
$$

\subsection{Pareto-optimal allocations}

We now want to characterize Pareto-optimal allocations and prove the equivalence between the properties 2. and 3. of the previous definition, thus generalizing Proposition 2.4. To do so, as in the standard setting described in Paragraph 2.1, the two following additional requirements have to be imposed on $U=U_{A}, U_{B}$ :

- $U$ satisfies the Non-Satiation Property $(\mathfrak{N} S)$, i.e. $\sup _{x \in \mathcal{L}} U(x)$ is not attained;

- $U$ is a concave functional:

$$
U(\alpha x+(1-\alpha) y) \geq \alpha U(x)+(1-\alpha) U(y), \quad \forall x, y \in \mathcal{L}, \alpha \in[0,1] .
$$

The first property simply states that there is no "best risk" in $\mathcal{L}$, while the second condition translates a broad notion of "risk aversion" of the associated preferences. As we have already noted, if $L$ is a space of random variables and $U(\cdot)=\mathbb{E} u(\cdot)$ is an expected utility, then the two assumptions above are certainly satisfied. 
Theorem 3.2 Assume that $U_{A}$ and $U_{B}$ satisfy the above two assumptions. An allocation is Pareto optimal if and only if it solves $(\lambda-S C)$ for some $\lambda>0$.

Proof. "If" part. If $\left(x^{A}, x^{B}\right) \in \mathcal{A}(\hat{x})$ is not Pareto optimal then there exists another allocation $\left(y^{A}, y^{B}\right) \in \mathcal{A}(\hat{x})$ such that $U_{A}\left(y^{A}\right) \geq U_{A}\left(x^{A}\right), U_{B}\left(y^{B}\right) \geq U_{B}\left(x^{B}\right)$ and at least one of the two inequalities is strict. This plainly yields $U_{A}\left(y^{A}\right)+$ $\lambda U_{B}\left(y^{B}\right)>U_{A}\left(x^{A}\right)+\lambda U_{B}\left(x^{B}\right) \forall \lambda>0$, i.e. $\left(x^{A}, x^{B}\right)$ cannot solve ( $\lambda$-SC).

"Only if" part. Let $\left(x_{*}^{A}, x_{*}^{B}\right) \in \mathcal{A}(\hat{x})$ be a Pareto optimal allocation and set

$$
\left(\theta_{*}^{A}, \theta_{*}^{B}\right) \triangleq\left(U_{A}\left(x_{*}^{A}\right), U_{B}\left(x_{*}^{B}\right)\right) \in \mathbb{R}^{2} .
$$

Then, define the three following subsets of $\mathbb{R}^{2}$ :

$$
\begin{aligned}
& \Theta \triangleq\left\{\left(\theta^{A}, \theta^{B}\right)=\left(U_{A}\left(x^{A}\right), U_{B}\left(x^{B}\right)\right):\left(x^{A}, x^{B}\right) \in \mathcal{A}(\hat{x})\right\}, \\
& \Pi \triangleq \Theta-\mathbb{R}_{+}^{2}=\left\{\left(\pi^{A}, \pi^{B}\right): \pi^{A} \leq \theta^{A}, \pi^{B} \leq \theta^{B} \text { for some }\left(\theta^{A}, \theta^{B}\right) \in \Theta\right\}, \\
& \Sigma \triangleq\left\{\left(\theta_{*}^{A}, \theta_{*}^{B}\right)\right\}+\mathbb{R}_{++}^{2}=\left\{\left(\sigma^{A}, \sigma^{B}\right): \sigma^{A} \geq \theta_{*}^{A}, \sigma^{B} \geq \theta_{*}^{B}\right\} \backslash\left\{\left(\theta_{*}^{A}, \theta_{*}^{B}\right)\right\} .
\end{aligned}
$$

We observe that

(a) П has non-empty (algebraic) interior as it contains, for instance, the open ball of radius 1 and center $\left(\theta_{*}^{A}-1, \theta_{*}^{B}-1\right)$.

(b) $\Pi$ and $\Sigma$ are disjoint: if $\Pi \cap \Sigma \neq \emptyset$, then it would easily follow that $\Theta \cap$ $\Sigma \neq \emptyset$ and this would be in contrast to the Pareto optimality of the allocation $\left(x_{*}^{A}, x_{*}^{B}\right)$.

(c) Both $\Pi$ and $\Sigma$ are convex: $\Sigma$ is convex by definition and $\Pi$ by concavity of the functions $U_{A}$ and $U_{B}$. Indeed, if $\left(\pi_{1}^{A}, \pi_{1}^{B}\right),\left(\pi_{2}^{A}, \pi_{2}^{B}\right) \in \Pi$, then

$$
\left\{\begin{array} { l } 
{ \pi _ { 1 } ^ { A } \leq U _ { A } ( x _ { 1 } ^ { A } ) } \\
{ \pi _ { 1 } ^ { B } \leq U _ { B } ( x _ { 1 } ^ { B } ) }
\end{array} \quad \left\{\begin{array}{l}
\pi_{2}^{A} \leq U_{A}\left(x_{2}^{A}\right) \\
\pi_{2}^{B} \leq U_{B}\left(x_{2}^{B}\right)
\end{array}\right.\right.
$$

for two allocations $\left(x_{1}^{A}, x_{1}^{B}\right)$ and $\left(x_{2}^{A}, x_{2}^{B}\right)$. From the concavity of $U_{A}$ and $U_{B}$, we obtain for any $\alpha \in(0,1)$ :$$
\left\{\begin{array}{l}
\alpha \pi_{1}^{A}+(1-\alpha) \pi_{2}^{A} \geq \alpha U_{A}\left(x_{1}^{A}\right)+(1-\alpha) U_{A}\left(x_{2}^{A}\right) \geq U_{A}\left(\alpha x_{1}^{A}+(1-\alpha) x_{2}^{A}\right), \\
\alpha \pi_{1}^{B}+(1-\alpha) \pi_{2}^{B} \geq \alpha U_{B}\left(x_{2}^{B}\right)+(1-\alpha) U_{B}\left(x_{2}^{B}\right) \geq U_{B}\left(\alpha x_{2}^{B}+(1-\alpha) x_{2}^{B}\right) .
\end{array}\right.
$$

Since $\left(\alpha x_{1}^{A}+(1-\alpha) x_{2}^{A}, \alpha x_{1}^{B}+(1-\alpha) x_{2}^{B}\right) \in \mathcal{A}(\hat{x})$, it follows that

$$
\left(\alpha \pi_{1}^{A}+(1-\alpha) \pi_{2}^{A}, \alpha \pi_{1}^{B}+(1-\alpha) \pi_{2}^{B}\right) \in \Pi,
$$

i.e. $\Pi$ is convex. 
We may then apply the algebraic Hahn-Banach Theorem (see for instance Aliprantis and Border (1999), Theorem 5.46) to the couple of sets $\Pi$ and $\Sigma$ and state the existence of a pair $\left(a^{A}, a^{B}\right) \in \mathbb{R}^{2} \backslash\{(0,0)\}$ such that

$$
a^{A} \pi^{A}+a^{B} \pi^{B} \leq a^{A} \sigma^{A}+a^{B} \sigma^{B}, \forall\left(\pi^{A}, \pi^{B}\right) \in \Pi,\left(\sigma^{A}, \sigma^{B}\right) \in \Sigma .
$$

Since $\left(\theta_{*}^{A}, \theta_{*}^{B}\right) \in \Pi$ and both $\left(\theta_{*}^{A}+1, \theta_{*}^{B}\right),\left(\theta_{*}^{A}, \theta_{*}^{B}+1\right) \in \Sigma$, it follows from the previous inequality that $a^{A}, a^{B} \geq 0$. As $\left(a^{A}, a^{B}\right) \neq(0,0)$, at least one of the two inequalities has to be strict: without any loss of generality we assume that $a^{A}>0$. For any $\varepsilon>0,\left(\theta_{*}^{A}+\varepsilon, \theta_{*}^{B}\right) \in \Sigma$ and therefore

$$
a^{A} \theta^{A}+a^{B} \theta^{B} \leq a^{A} \theta_{*}^{A}+a^{B} \theta_{*}^{B}+a^{A} \varepsilon, \quad \forall\left(\theta^{A}, \theta^{B}\right) \in \Theta .
$$

Letting $\varepsilon \backslash 0$ we obtain:

$$
a^{A} \theta^{A}+a^{B} \theta^{B} \leq a^{A} \theta_{*}^{A}+a^{B} \theta_{*}^{B}, \forall\left(\theta^{A}, \theta^{B}\right) \in \Theta,
$$

i.e. $\left(x_{*}^{A}, x_{*}^{B}\right)$ solves the optimization program:

$$
\sup _{\left(x^{A}, x^{B}\right) \in \mathcal{A}(\hat{x})}\left\{a^{A} U_{A}\left(x^{A}\right)+a^{B} U_{B}\left(x^{B}\right)\right\} .
$$

Let us now prove that $a^{B}>0$. If $a^{B}=0$ then, reminding that $\mathcal{A}(\hat{x})=\{(x, \hat{x}-x) \in$ $\left.\mathcal{L}^{2}: x \in \mathcal{L}\right\}$, the optimization program above would become:

$$
\sup _{\left(x^{A}, x^{B}\right) \in \mathcal{A}(\hat{x})} a^{A} U_{A}\left(x^{A}\right)=a^{A} \sup _{x \in \mathcal{L}} U_{A}(x),
$$

which has no solution in view of property $(\mathcal{N} \mathcal{S})$ for $U_{A}$. By setting $\lambda=a^{B} / a^{A}>0$ we readily obtain the desired result.

Remark 3.3 The above Theorem is related to the existing literature and can be seen as a generalization of various results. More precisely,

i) it extends Proposition 2.4 and Theorem 2.8, but also some more recent results on convex risk measures (see Barrieu and El Karoui (2005 and 2006)) or on monetary utility functions (see Acciaio (2007) or Jouini, Schachemayer and Touzi (2008)). Moreover, while the previous results refer to some specific preference functionals defined on some spaces of random variables, Theorem 3.2 is more general as it holds for any concave preference functionals defined on a general vector space. Therefore, its scope is wider, and in particular it can be applied to a multi-period setting as we will see in the next section.

ii) Our result can also be seen as a generalization of Proposition 10C of Duffie (1996) in two different aspects: the set of conditions and the space of risks that is considered. Indeed, while Duffie required as condition for the result to hold true the strict increasing monotonicity of the preference functional $U$, we ask 
for the weaker condition of Non-Satiation property (which is implied by the strict increasing monotonicity but does not imply it). Moreover, while Duffie considered as space of risks that of non-negative adapted square integrable processes, our result is obtained on a real vector space. His result is stated for $m \geq 2$ agents, but the extension of our result to $m$ agents is straightforward.

iii) There is a wide economic literature on the notion of Arrow-Debreu equilibrium. In particular, Mas-Colell (1986) gives (Theorem 2 therein) a necessary condition for an allocation to be Pareto optimal, interpreting the value of the sup-convolution of the utilities (multiplied by suitable positive weights) as a supporting price functional. This result, that can be viewed as an abstract KuhnTucker criterion, is surely linked to our Theorem 3.2 above. However there are some substantial differences. On the one hand, the framework Mas-Colell considers is in a sense more general as it involves an arbitrary (but finite) number of agents and distinguishes between consumers and producers, while we consider only two consumers. On the other hand, his setting is less general than ours as the space of commodities (our general vector space $\mathcal{L}$ ) is assumed to be an ordered locally convex vector space and the utility functionals are assumed to be affine and continuous. Moreover, in Mas-Colell (1986) the primary focus is in obtaining the existence of (linear and continuous) pricing functionals ensuring a general equilibrium, while in the present paper we are interested in the optimal allocation rules between two agents, with no particular interest in general equilibrium issues.

\section{Application to the multi-Period Setting}

We now consider a multi-period setting where the risks, i.e. uncertain future monetary cash flows are possibly spread over $N \geq 2$ different future dates and are not concentrated at a single maturity any longer. Introducing several possible dates is rather natural, as the different agents may have different investment time horizons, but also each agent may have a stream of risks, or various cash flows, occurring at different possible times. In these situations, considering oneperiod agreements is of course a possibility but the agents may prefer to sign a long-term agreement, or multi-period agreement, with another agent instead to doing a "roll-over" of a single period agreement with the counterpart and re-negotiating it at each time-step. Agents may also prefer to deal with the risk when it occurs rather than waiting for a further time horizon (which could have potentially no particular economic significance for them) as this would be the case when considering a unique possible risk exchange date in the future, corresponding to the furthest date in this study. This is especially true in the case of long-term risks, such as longevity risk. Exposed agents, such as pension funds, might prefer to receive some form of compensation periodically over a long period of time rather than wait for a far away maturity date to get compensated. 


\subsection{Notation and Pareto optimal allocations}

There are now $N \geq 2$ future times $\left(t_{n}\right)_{1 \leq n \leq N}$, with $t_{n}>t_{n-1}$ and $t_{1}>0$. We naturally add to the probability space $(\Omega, \mathcal{F}, \mathbb{P})$ a filtration $\mathbb{F}=\left(\mathcal{F}_{n}\right)_{n \leq N}$, where $\mathcal{F}_{n} \subset \mathcal{F}$ is the information up to time $t_{n}$. For any $n$, let $L_{n}^{0} \triangleq L^{0}\left(\Omega, \mathcal{F}_{n}, \mathbb{P}\right)$ be the space of $\mathcal{F}_{n}$-measurable random variables, describing the risks occurring at time $t_{n}$. The subspaces of integrable (resp. bounded) random variables will be denoted with $L_{n}^{1}$ (resp. $\left.L_{n}^{\infty}\right)$. More precisely, $X_{n}$ is the cumulated risk over $\left(t_{n-1}, t_{n}\right]$. There is no overlapping between the different risks and the first component of a risk stream occurs at time $t_{1}$. The space of all possible risk streams is then

$$
\mathbf{L}^{0} \triangleq\left\{\mathbf{X}=\left(X_{n}\right)_{1 \leq n \leq N}: X_{n} \in L_{n}^{0}, \forall n\right\} .
$$

In a similar way we denote the subspace of integrable (resp. bounded) risk streams as $\mathbf{L}^{1}$ (resp. as $\mathbf{L}^{\infty}$ ). Finally, $\mathbf{0}=(0, \ldots, 0)$ will denote the null process and, $\mathbf{1}_{n}$ is the process having 1 in the $n$-th place (for a fixed $n \leq N$ ) and 0 otherwise.

Let $\mathbf{L} \subseteq \mathbf{L}^{0}$ be a generic subspace that collects all the risk streams that interest. Note that $\mathbf{L}$ is a particular example of the general real vector space $\mathcal{L}$ introduced in the last section. Therefore, we can use the general definitions previously introduced. More precisely, the agents now initially face the risk streams

$$
\widehat{\mathbf{X}}^{A}=\left(\widehat{X}_{n}^{A}\right)_{1 \leq n \leq N} \in \mathbf{L} \quad \text { and } \quad \widehat{\mathbf{X}}^{B}=\left(\widehat{X}_{n}^{B}\right)_{1 \leq n \leq N} \in \mathbf{L} .
$$

The aggregate risk stream is $\widehat{\mathbf{X}}=\widehat{\mathbf{X}}^{A}+\widehat{\mathbf{X}}^{B}$, or explicitly:

$$
\widehat{X}_{n}=\widehat{X}_{n}^{A}+\widehat{X}_{n}^{B} \quad \forall n .
$$

The set of all possible allocations is therefore

$$
\mathcal{A}(\widehat{\mathbf{X}})=\left\{\left(\mathbf{X}^{A}, \mathbf{X}^{B}\right) \in \mathbf{L} \times \mathbf{L}: \mathbf{X}^{A}+\mathbf{X}^{B}=\widehat{\mathbf{X}}\right\} .
$$

If the preferences of the agents over risk streams in $\mathbf{L}$ are described by two preference functionals $U_{A}, U_{B}: \mathbf{L} \rightarrow \mathbb{R}$, then an allocation $\left(\mathbf{X}^{A}, \mathbf{X}^{B}\right) \in \mathcal{A}(\widehat{\mathbf{X}})$ is Pareto optimal if for any other allocation $\left(\mathbf{Y}^{A}, \mathbf{Y}^{B}\right) \in \mathcal{A}(\widehat{\mathbf{X}})$ it holds

$$
\begin{aligned}
& U_{A}\left(\mathbf{Y}^{A}\right)>U_{A}\left(\mathbf{X}^{A}\right) \Longrightarrow U_{B}\left(\mathbf{Y}^{B}\right)<U_{B}\left(\mathbf{X}^{B}\right), \\
& U_{B}\left(\mathbf{Y}^{B}\right)>U_{B}\left(\mathbf{X}^{B}\right) \Longrightarrow U_{A}\left(\mathbf{Y}^{A}\right)<U_{A}\left(\mathbf{X}^{A}\right) .
\end{aligned}
$$

As this multi-period setting is a particular case of the general framework previously described in Section 3, we immediately obtain the following corollary of the general Theorem 3.2:

Corollary 4.1 Assume that both functionals $U_{A}$ and $U_{B}$ are concave and satisfy the Non-Satiation Property ( $\mathcal{N} S$ ). Then an allocation $\left(\mathbf{X}^{A}, \mathbf{X}^{B}\right) \in \mathcal{A}(\widehat{\mathbf{X}})$ is Pareto optimal if and only if it solves, for some $\lambda>0$, the $(\lambda-S C)$ problem: 


$$
\left[U_{A} \square \lambda U_{B}\right](\widehat{\mathbf{X}}) \triangleq \sup _{\left(\mathbf{Y}^{A}, \mathbf{Y}^{B}\right) \in \mathcal{A}(\hat{\mathbf{X}})}\left\{U_{A}\left(\mathbf{Y}^{A}\right)+\lambda U_{B}\left(\mathbf{Y}^{B}\right)\right\} .
$$

The corollary gives a partial extension of the Theorem 2.8. More precisely the equivalence between statements 1 . and 2 . is extended here to the multi-period setting. However, to obtain a full generalization, an equivalent of statement 3. has to be found. To do so, we specify a class of preference functionals defined on $\mathbf{L}$ and consider expected utilities for processes.

\subsection{Expected utilities for processes}

In the rest of the paper, we are interested in further characterizing Pareto optimal allocations and allocation rules under some particular choices for the preference functionals of both agents. More precisely, we introduce a fairly general class of such functionals, by considering those which can be represented as expected utilities for processes, and derive the related necessary and sufficient conditions for Pareto optimality. In the later sections we illustrate these results to some relevant cases.

Definition 4.2 A utility function for processes is a differentiable map $w: \mathbb{R}^{N} \rightarrow \mathbb{R}$ that is strictly increasing in at least one variable and concave. We further assume that all its partial derivatives are continuous.

Definition 4.3 An expected utility for processes is any preference functional $U$ : $\mathbf{L} \rightarrow \mathbb{R}$ of the form:

$$
U\left(X_{1}, \ldots, X_{N}\right) \triangleq \mathbb{E} w\left(X_{1}, \ldots, X_{N}\right),
$$

for some utility function for processes.

Any expected utility for processes is plainly concave as $w$ is concave. It also satisfies the Non-Satiation Property $(\mathfrak{N} S)$ : indeed if $w$ is strictly increasing in $x_{n}$ (such an index $n$ exists by definition), then $U\left(X_{1}, \ldots, X_{N}\right)<U\left(X_{1}, \ldots, X_{n}+\right.$ $1, \ldots, X_{N}$ ) for any $\mathbf{X} \in \mathbf{L}$. For similar reasons as in the one-period case (see the discussion after Definition 2.5) we do not further specify the space $\mathbf{L}$, but we assume throughout this section that $\mathbf{L}^{\infty} \subseteq \mathbf{L}$.

Assume now that the preferences of both agents are represented by an expected utility for processes, i.e. $U_{A}(\mathbf{X})=\mathbb{E} w_{A}(\mathbf{X})$ and $U_{B}(\mathbf{X})=\mathbb{E} w_{B}(\mathbf{X})$ for two utility functions for processes $w_{A}$ and $w_{B}$.

In order to characterize Pareto optimal allocations, we will need the following straightforward generalization of Lemma 2.7 that we state here without the proof.

Lemma 4.4 Let $w$ be a utility function for processes and fix $n \in\{1, \ldots, N\}$. If $\mathbf{X} \in \mathbf{L}$ and $S \in \mathcal{F}_{n}$ are such that

$$
I_{S} \frac{\partial w}{\partial x_{n}}\left(\mathbf{X}-\varepsilon \mathbf{1}_{n}\right) \in L^{1}
$$


for some $\varepsilon>0$, then the map $h(\alpha) \triangleq \mathbb{E} w\left(X_{1}, \ldots, X_{n}+\alpha I_{S}, \ldots, X_{N}\right), \alpha \in \mathbb{R}$, is differentiable at $\alpha=0$ and

$$
h^{\prime}(0)=\mathbb{E}\left[I_{S} \frac{\partial w}{\partial x_{n}}(\mathbf{X})\right]
$$

It is possible to obtain necessary and/or sufficient conditions for Pareto optimality, partly generalizing Theorem 2.8. However, contrary to Theorem 2.8, some additional conditions are needed and everything depends on the considered space of risks, as we will see in the three following paragraphs. The underlying logic of the different results (Theorems 4.5, 4.6 and 4.7) is however very similar to that of Theorem 2.8. In particular, the interpretation in terms of pricing measures is still valid: the agents should agree on the pricing measure. However, because of the multi-period framework, this condition has to hold true at any time $n(n=1,2, \ldots N)$, taking into account the arrival of new information (which is expressed in terms of measurability, or conditional expected value of the marginal preferences). Moreover, since the decision of the allocation of risk is taken at time 0 but implies risk exchanges at various times $n=1, \ldots N$ in the future, the relationship between the beliefs of the agents for the optimum has to be robust over time (which is expressed by the constant $\lambda$ ) to ensure the existence of an initial agreement between them.

\subsubsection{Pareto optimality for bounded risks}

We first consider the question of Pareto optimality for bounded allocations, i.e. when $\mathbf{L}=\mathbf{L}^{\infty}$. In this case it is possible to state a necessary and sufficient condition for Pareto optimality, which fully generalizes the one-period result.

Theorem 4.5 An allocation $\left(\mathbf{X}^{A}, \mathbf{X}^{B}\right) \in \mathbf{L}^{\infty} \times \mathbf{L}^{\infty}$ is Pareto optimal if and only if there exists a constant $\lambda>0$ such that for any $n=1, \ldots N$ :

$$
\mathbb{E}\left[\frac{\partial w_{A}}{\partial x_{n}}\left(\mathbf{X}^{A}\right) \mid \mathcal{F}_{n}\right]=\lambda \mathbb{E}\left[\frac{\partial w_{B}}{\partial x_{n}}\left(\mathbf{X}^{B}\right) \mid \mathcal{F}_{n}\right] .
$$

Proof. "Only if" part. The proof is similar to that of Theorem $2.8(2 \Rightarrow 3)$, the idea being to focus on the $n$-th component of the allocation (for any fixed $n$ ). We report it for completeness. Since both preference functionals $U_{A}$ and $U_{B}$ are concave and satisfy the Non-Satiation Property $(\mathcal{N} S)$, we can apply Corollary 4.1. Therefore $\left(\mathbf{X}^{A}, \mathbf{X}^{B}\right)$ solves $(\lambda$-SC) for some $\lambda>0$. Let us fix $n \in\{1, \ldots, N\}$ and set for the sake of simplicity

$$
W_{A} \triangleq \mathbb{E}\left[\frac{\partial w_{A}}{\partial x_{n}}\left(\mathbf{X}^{A}\right) \mid \mathcal{F}_{n}\right] \text { and } W_{B} \triangleq \mathbb{E}\left[\frac{\partial w_{B}}{\partial x_{n}}\left(\mathbf{X}^{B}\right) \mid \mathcal{F}_{n}\right]
$$


By assumption both $W_{A}$ and $W_{B}$ are bounded. For any bounded interval $C \subset \mathbb{R}$ and any $\alpha \in \mathbb{R}$, we can build the following admissible allocation:

$$
\left\{\begin{array}{l}
\mathbf{Y}^{A}(\alpha, C)=\left(X_{1}^{A}, \ldots, X_{n}^{A}+\alpha I_{S(C)}, \ldots, X_{N}^{A}\right) \\
\mathbf{Y}^{B}(\alpha, C)=\left(X_{1}^{B}, \ldots, X_{n}^{B}-\alpha I_{S(C)}, \ldots, X_{N}^{B}\right),
\end{array}\right.
$$

where

$$
S(C) \triangleq\left\{W_{A}-\lambda W_{B} \in C\right\} \in \mathcal{F}_{n} .
$$

Since $\left(\mathbf{X}^{A}, \mathbf{X}^{B}\right)$ solves $(\lambda$-SC), for any fixed $C$ the map:

$$
f(\alpha) \triangleq \mathbb{E} w_{A}\left(\mathbf{Y}^{A}(\alpha, C)\right)+\lambda \mathbb{E} w_{B}\left(\mathbf{Y}^{B}(\alpha, C)\right), \alpha \in \mathbb{R} .
$$

clearly attains a maximum at $\alpha=0$. Since $\mathbf{X}^{A}$ is bounded and the partial derivatives of $w_{A}$ are continuous, it follows that $\frac{\partial w_{A}}{\partial x_{n}}\left(\mathbf{X}^{A}-\varepsilon \mathbf{1}_{n}\right)$ is bounded for some (actually, any) $\varepsilon>0$. Therefore we can apply Lemma 4.4, obtaining:

$$
f_{A}^{\prime}(0)=\mathbb{E}\left[I_{S(C)} \frac{\partial w_{A}}{\partial x_{n}}\left(\mathbf{X}^{A}\right)\right]=\mathbb{E}\left[I_{S(C)} W_{A}\right]
$$

where $f_{A}(\alpha) \triangleq \mathbb{E} w_{A}\left(\mathbf{Y}^{A}(\alpha, C)\right)$. In a similar way we prove that $f_{B}^{\prime}(0)=\mathbb{E}\left[I_{S(C)} W_{B}\right]$ where $f_{B}(\alpha) \triangleq \mathbb{E} w_{B}\left(\mathbf{Y}^{B}(\alpha, C)\right)$. As a consequence the map $f$ is differentiable at $\alpha=0$ and $f^{\prime}(0)=\mathbb{E}\left[I_{S(C)}\left(W_{A}-\lambda W_{B}\right)\right]$. The first order condition for a maximum is then:

$$
\mathbb{E}\left[I_{\left\{W_{A}-\lambda W_{B} \in C\right\}}\left(W_{A}-\lambda W_{B}\right)\right]=0 \quad \forall C .
$$

As $C$ is a generic bounded interval, we can conclude that $W_{A}-\lambda W_{B}$ vanishes P-a.s. and we obtain (6).

"If" part. Let $\left(\mathbf{X}^{A}, \mathbf{X}^{B}\right) \in \mathcal{A}(\widehat{\mathbf{X}})$ be an allocation satisfying (6) and let us consider another possible allocation $\left(\mathbf{Y}^{A}, \mathbf{Y}^{B}\right)$. Since both $w_{A}$ and $w_{B}$ are concave, we have:

$$
w_{A}\left(\mathbf{Y}^{A}\right) \leq w_{A}\left(\mathbf{X}^{A}\right)+\sum_{n=1}^{N}\left(Y_{n}^{A}-X_{n}^{A}\right) \frac{\partial w_{A}}{\partial x_{n}}\left(\mathbf{X}^{A}\right)
$$

and

$$
w_{B}\left(\mathbf{Y}^{B}\right) \leq w_{B}\left(\mathbf{X}^{B}\right)+\sum_{n=1}^{N}\left(Y_{n}^{B}-X_{n}^{B}\right) \frac{\partial w_{B}}{\partial x_{n}}\left(\mathbf{X}^{B}\right) .
$$

Multiplying the second inequality by $\lambda>0$ and adding it to the first one we obtain:

$$
w_{A}\left(\mathbf{Y}^{A}\right)+\lambda w_{B}\left(\mathbf{Y}^{B}\right) \leq w_{A}\left(\mathbf{X}^{A}\right)+\lambda w_{B}\left(\mathbf{X}^{B}\right)+\Psi,
$$


where $\Psi=\sum_{n=1}^{N}\left\lfloor\left(Y_{n}^{A}-X_{n}^{A}\right) \frac{\partial w_{A}}{\partial x_{n}}\left(\mathbf{X}^{A}\right)+\lambda\left(Y_{n}^{B}-X_{n}^{B}\right) \frac{\partial w_{B}}{\partial x_{n}}\left(\mathbf{X}^{B}\right)\right\rfloor$.

As every random variable involved in the definition of $\Psi$ is bounded, we can compute its expectation:

$$
\begin{aligned}
\mathbb{E} \Psi & =\sum_{n=1}^{N} \mathbb{E}\left\{\mathbb{E}\left[\left(Y_{n}^{A}-X_{n}^{A}\right) \frac{\partial w_{A}}{\partial x_{n}}\left(\mathbf{X}^{A}\right)+\lambda\left(Y_{n}^{B}-X_{n}^{B}\right) \frac{\partial w_{B}}{\partial x_{n}}\left(\mathbf{X}^{B}\right) \mid \mathcal{F}_{n}\right]\right\} \\
& =\sum_{n=1}^{N} \mathbb{E}\left\{\left(Y_{n}^{A}-X_{n}^{A}\right) \mathbb{E}\left[\frac{\partial w_{A}}{\partial x_{n}}\left(\mathbf{X}^{A}\right) \mid \mathcal{F}_{n}\right]+\left(Y_{n}^{B}-X_{n}^{B}\right) \lambda \mathbb{E}\left[\frac{\partial w_{B}}{\partial x_{n}}\left(\mathbf{X}^{B}\right) \mid \mathcal{F}_{n}\right]\right\} \\
& =\sum_{n=1}^{N} \mathbb{E}\left\{\left(Y_{n}^{A}-X_{n}^{A}+Y_{n}^{B}-X_{n}^{B}\right) \mathbb{E}\left[\frac{\partial w_{A}}{\partial x_{n}}\left(\mathbf{X}^{A}\right) \mid \mathcal{F}_{n}\right]\right\}
\end{aligned}
$$

The first equality is the tower property of conditional expectations, the second one follows from the fact that, for any $n, Y_{n}^{A}-X_{n}^{A}$ and $Y_{n}^{B}-X_{n}^{B}$ are bounded, while the last one is a direct consequence of (6). Since, for any $n, X_{n}^{A}+X_{n}^{B}=$ $\widehat{X}_{n}=Y_{n}^{A}+Y_{n}^{B}$ it readily follows $\mathbb{E} \Psi=0$. Therefore, taking the expected value on both sides of the inequality (9), we obtain

$$
U_{A}\left(\mathbf{Y}^{A}\right)+\lambda U_{B}\left(\mathbf{Y}^{B}\right) \leq U_{A}\left(\mathbf{X}^{A}\right)+\lambda U_{B}\left(\mathbf{X}^{B}\right)
$$

and we conclude that $\left(\mathbf{X}^{A}, \mathbf{X}^{B}\right)$ solves $(\lambda$-SC), hence it is Pareto optimal.

\subsubsection{Pareto optimality for general risks}

In this section, we drop the hypothesis of bounded risks and simply consider $\mathbf{L}$ such that $\mathbf{L}^{\infty} \subseteq \mathbf{L}$. We can derive some conditions for Pareto optimality under various assumptions on the expected utility for processes we consider.

Pareto optimality under a measurability condition. Let us focus on the expected utilities for processes $w$ such that:

$$
\frac{\partial w}{\partial x_{n}} \text { depends only on } x_{n}, \forall n \text {. }
$$

Note that this condition is trivially satisfied in the one-period framework. In the present multi-period setting, it is satisfied in particular by all time-additive preference functionals (see Subsection 5.1). Note that this property implies that for any allocation $\left(\mathbf{X}^{A}, \mathbf{X}^{B}\right)$,

$$
\frac{\partial w_{i}}{\partial x_{n}}\left(\mathbf{X}^{i}\right) \text { is } \mathcal{F}_{n} \text {-measurable, } \quad \forall n, i=A, B
$$

and this is the key property to prove the following result. 
Theorem 4.6 Assume that $w_{A}$ and $w_{B}$ satisfy the measurability condition (10). Then an allocation $\left(\mathbf{X}^{A}, \mathbf{X}^{B}\right)$ is Pareto optimal if and only if there exists a constant $\lambda>0$ such that, for any $n=1, \ldots, N$

$$
\frac{\partial w_{A}}{\partial x_{n}}\left(\mathbf{X}^{A}\right)=\lambda \frac{\partial w_{B}}{\partial x_{n}}\left(\mathbf{X}^{B}\right) .
$$

Proof. The proof is similar to that of Theorem 4.5, and therefore, we use the same notation. However, due to the difference in the hypotheses we make, there are some important modifications that we highlight below.

In the "only if" part we can set, for a fixed $n$,

$$
W_{A} \triangleq \frac{\partial w_{A}}{\partial x_{n}}\left(\mathbf{X}^{A}\right) \in \mathcal{F}_{n} \quad \text { and } \quad W_{B} \triangleq \frac{\partial w_{B}}{\partial x_{n}}\left(\mathbf{X}^{B}\right) \in \mathcal{F}_{n},
$$

instead of considering the conditional expected values, because of the measurability condition. Define, for a bounded interval $C$ and $k \in \mathbb{N}$

$$
S(C, k) \triangleq\left\{\left|W_{A}\right|,\left|W_{B}\right| \leq k, W_{A}-\lambda W_{B} \in C\right\},
$$

and the risk allocations

$$
\left\{\begin{array}{l}
\mathbf{Y}^{A}(\alpha, C, k)=\left(X_{1}^{A}, \ldots, X_{n}^{A}+\alpha I_{S(C)}, \ldots, X_{N}^{A}\right) \\
\mathbf{Y}^{B}(\alpha, C, k)=\left(X_{1}^{B}, \ldots, X_{n}^{B}-\alpha I_{S(C)}, \ldots, X_{N}^{B}\right) .
\end{array}\right.
$$

Proceeding as in Theorem 2.8 and using Lemma 4.4 we obtain the first order conditions:

$$
\mathbb{E}\left[I_{S(C, k)}\left(W_{A}-\lambda W_{B}\right)\right]=0, \forall C, k .
$$

Through the same limiting procedure in $k$ used in Theorem 2.8, we then obtain:

$$
\mathbb{E}\left[I_{S(C)}\left(W_{A}-\lambda W_{B}\right)\right]=0, \forall C,
$$

where, as before, $S(C) \triangleq\left\{W_{A}-\lambda W_{B} \in C\right\} \in \mathcal{F}_{n}$. We conclude that $W_{A}-\lambda W_{B}=0$.

The proof of the "if part" is very similar to the corresponding part in Theorem 4.5. However, using (12), it is possible to prove that the random variable $\Psi$ appearing in (9) vanishes $\mathbb{P}$-a.s., and not only after taking the expectation.

Pareto optimality under an integrability condition. A preference functional which is not time-additive in general does not satisfy the measurability condition (11) (see Subsection 5.2). Therefore, the last Theorem does not necessarily hold true in these cases. It is however still possible to prove a necessary condition 
for Pareto optimality of an allocation $\left(\mathbf{X}^{A}, \mathbf{X}^{B}\right)$ under the following integrability condition:

$$
\frac{\partial w_{i}}{\partial x_{n}}\left(\mathbf{X}^{i}\right) \in L^{1}, \forall n, i=A, B
$$

Theorem 4.7 Let $\left(\mathbf{X}^{A}, \mathbf{X}^{B}\right)$ be an allocation satisfying the integrability condition (13). If it is Pareto optimal then there exists a constant $\lambda>0$ such that

$$
\mathbb{E}\left[\frac{\partial w_{A}}{\partial x_{n}}\left(\mathbf{X}^{A}\right) \mid \mathcal{F}_{n}\right]=\lambda \mathbb{E}\left[\frac{\partial w_{B}}{\partial x_{n}}\left(\mathbf{X}^{B}\right) \mid \mathcal{F}_{n}\right], \quad \forall n
$$

Proof. The proof of this result is identical to the corresponding "only if" part in Theorem 4.6. The only difference is that we now set $W_{A} \triangleq \mathbb{E}\left[\frac{\partial w_{A}}{\partial x_{n}}\left(\mathbf{X}^{A}\right) \mid \mathcal{F}_{n}\right]$ and $W_{B} \triangleq \mathbb{E}\left[\frac{\partial w_{B}}{\partial x_{n}}\left(\mathbf{X}^{B}\right) \mid \mathcal{F}_{n}\right]$, as in Theorem 4.5.

Remark 4.8 In this case it is not possible to prove that the condition (14) is also sufficient for Pareto optimality. Indeed, in this case we are no longer able to write:

$$
\mathbb{E}\left[X_{n} \frac{\partial w}{\partial x_{n}}(\mathbf{X}) \mid \mathcal{F}_{n}\right]=X_{n} \mathbb{E}\left[\frac{\partial w}{\partial x_{n}}(\mathbf{X}) \mid \mathcal{F}_{n}\right],
$$

as $X_{n}$ is not bounded and therefore the left hand side may not be well defined. This fact prevents us from using the same argument for the "if" part in Theorem 4.5 .

\section{SOME RELEVANT EXAMPLES}

We now apply the previous results to some relevant examples of expected utilities for processes. We focus only on bounded allocations, in order to fully exploit the results of the previous section. In other words we set $\mathbf{L}=\mathbf{L}^{\infty}$ throughout this section, and Theorem 4.5 holds true. Also, for the sake of clarity we shall state the results only in a 2-period model (i.e. $N=2$ ). The main focus of this study is really on passing from one to two periods. The extensions of the results to the case $(N \geq 3)$ is straightforward, but sometimes heavy in terms of formulae. As a consequence we will present general formulations of the results only in the exponential utility framework, when they are still sufficiently clear.

In the next paragraph we consider time-additive preference functionals, both in their classical (i.e. "state-independent") formulation and in a "state-dependent" one, in which the subjective decay factors are allowed to depend on past risks. In the subsequent paragraph, a simple example of a non time-additive functional is treated. Finally, in the last paragraph we illustrate these results by considering exponential utility functions. We will see in particular that in the 
example we consider, every Pareto optimal allocation can be written in terms of an allocation rule extending the former Wyler's results (Proposition 2.14) to a multi-period setting.

\subsection{Functionals of time-additive type}

\subsubsection{The classical "state-independent" case}

A (classical) time-additive preference functional for processes comes, by definition, in the form

$$
U(X)=\sum_{n=1}^{N} \beta^{n-1} \mathbb{E} u\left(X_{n}\right)=\mathbb{E} u\left(X_{1}\right)+\beta \mathbb{E} u\left(X_{2}\right)+\ldots+\beta^{N-1} \mathbb{E} u\left(X_{N}\right),
$$

where $u$ is a (classical) utility function and $\beta>0$ a constant called subjective decay factor. Usually $\beta \in(0,1]$ and is related to a discount factor involving real interest rates. However the interpretation of $\beta$ is not really our focus here, and the result below holds for $\beta>1$ as well. The functional $U$ is an expected utility for processes as $U(\mathbf{X})=\mathbb{E} w(\mathbf{X})$, where

$$
w\left(x_{1}, \ldots, x_{N}\right)=\sum_{n=1}^{N} \beta^{n-1} u\left(x_{n}\right),
$$

and this last map is clearly concave and strictly increasing in each variable.

We now focus on a 2-period model and assume that the agents $A$ and $B$ have preference functionals of the following type

$$
U_{i}\left(X_{1}, X_{2}\right) \triangleq \mathbb{E} u_{i}\left(X_{1}\right)+\beta_{i} \mathbb{E} u_{i}\left(X_{2}\right), i=A, B
$$

for two utility functions $u_{A}$ and $u_{B}$ and two decay factors $\beta_{A}, \beta_{B}>0$.

Proposition 5.1 Under the above specification for $U_{A}$ and $U_{B}$, the following statements are equivalent:

1. The allocation $\left(X_{1}^{A}, X_{2}^{A}\right)$ is Pareto optimal;

2. $X_{1}^{A}=\phi_{1}\left(\widehat{X}_{1}\right)$ and $X_{2}^{A}=\phi_{2}\left(\widehat{X}_{2}\right)$, where the maps $\phi_{1}$ and $\phi_{2}$ satisfy, for some constant $\lambda>0$,

$$
\begin{gathered}
u_{A}^{\prime}\left(\phi_{1}\left(\widehat{X}_{1}\right)\right)=\lambda u_{B}^{\prime}\left(\widehat{X}_{1}-\phi_{1}\left(\widehat{X}_{1}\right)\right), \\
\beta_{A} u_{A}^{\prime}\left(\phi_{2}\left(\widehat{X}_{2}\right)\right)=\lambda \beta_{B} u_{B}^{\prime}\left(\widehat{X}_{2}-\phi_{2}\left(\widehat{X}_{2}\right)\right) ;
\end{gathered}
$$

3. $X_{1}^{A}=\phi_{1}\left(\widehat{X}_{1}\right)$ and $X_{2}^{A}=\phi_{2}\left(\widehat{X}_{2}\right)$, where the maps $\phi_{1}$ and $\phi_{2}$ are differentiable and solve for any $\left(x_{1}, x_{2}\right) \in \mathbb{R}^{2}$

$$
\phi_{1}^{\prime}\left(x_{1}\right)=\frac{R A_{B}\left(x_{1}-\phi_{1}\left(x_{1}\right)\right)}{R A_{A}\left(\phi_{1}\left(x_{1}\right)\right)+R A_{B}\left(x_{1}-\phi_{1}\left(x_{1}\right)\right)},
$$




$$
\phi_{2}^{\prime}\left(x_{2}\right)=\frac{R A_{B}\left(x_{2}-\phi_{2}\left(x_{2}\right)\right)}{R A_{A}\left(\phi_{2}\left(x_{2}\right)\right)+R A_{B}\left(x_{2}-\phi_{2}\left(x_{2}\right)\right)},
$$

where $R A_{i}$ denotes the Arrow-Pratt coefficient of absolute risk aversion agent $i(i=A, B)$.

Proof. The equivalence $1 . \Leftrightarrow 2$. is partly a consequence of the previous Theorem 4.5. Indeed, we know that an allocation $\left(\mathbf{X}^{A}, \mathbf{X}^{B}\right)$ is Pareto optimal if and only if

$$
\begin{aligned}
u_{A}^{\prime}\left(X_{1}^{A}\right) & =\lambda u_{B}^{\prime}\left(X_{1}^{B}\right), \\
\beta_{A} u_{A}^{\prime}\left(X_{2}^{A}\right) & =\lambda \beta_{B} u_{B}^{\prime}\left(X_{2}^{B}\right),
\end{aligned}
$$

for some $\lambda>0$.

It remains to prove that the optimal allocations are pool rearrangements. Proposition 2.14 ensures that $X_{1}^{A}=\phi_{1}\left(\widehat{X}_{1}\right)$ and $X_{2}^{A}=\phi_{2}\left(\widehat{X}_{2}\right)$ where $\phi_{1}$ and $\phi_{2}$ satisfy (16). Moreover, since the utility functions $u_{A}$ and $u_{B}$ are twice differentiable by assumption, $\phi$ and $\phi_{2}$ are also differentiable and solve the system (18).

Note that the appropriate constant $\lambda$ is given in this case by $\lambda=\frac{u_{A}^{\prime}\left(\phi_{1}(0)\right)}{u_{B}^{\prime}\left(-\phi_{1}(0)\right)}=$ $\frac{\beta_{A} u_{A}^{\prime}\left(\phi_{2}(0)\right)}{\beta_{B} u_{B}^{\prime}\left(-\phi_{2}(0)\right)}$.

Remark 5.1 In this setting, if $\left(\mathbf{X}^{A}, \mathbf{X}^{B}\right)$ is a Pareto optimal allocation, then

$$
\frac{u_{A}^{\prime}\left(X_{1}^{A}\right)}{u_{B}^{\prime}\left(X_{1}^{B}\right)}=\lambda \text { and } \frac{u_{A}^{\prime}\left(X_{2}^{A}\right)}{u_{B}^{\prime}\left(X_{2}^{B}\right)}=\lambda \frac{\beta_{B}}{\beta_{A}} \text {. }
$$

Therefore, at each time, the ratio of the agents' marginal utilities is constant and independent of the risks. Moreover, the evolution of the marginal utility ratio over time is fully characterized by the ratio of the decay factors and it does not depend on the risks.

Remark 5.2 At any time, any Pareto optimal allocation depends only on the total aggregated risk of that particular time. This characterization is similar to that of Borch in the one-period setting for expected utility (see Proposition 2.14). As a consequence, there is no intertemporal exchange of risk between the two agents. This result appears to be quite natural given the type of the utility functionals considered. The assessment of the risk is indeed specific to each time period and the aggregation comes only after the assessment.

\subsubsection{State-dependent case}

We now consider a generalization of the previous model in which the decay factor $\beta>0$ is allowed to depend on the risk experienced in the past. In other words, in a 2-period setting we replace the characterization (15) with 


$$
U_{i}\left(X_{1}, X_{2}\right) \triangleq \mathbb{E}\left[u_{i}\left(X_{1}\right)+\beta_{i}\left(X_{1}\right) u_{i}\left(X_{2}\right)\right], i=A, B
$$

where $u_{A}$ and $u_{B}$ are two utility functions and $\beta_{A}, \beta_{B}: \mathbb{R} \rightarrow(0, \infty)$ are two twice differentiable maps describing how the decay factors depend on the past risks. For instance, a natural dependency structure may be a decreasing relationship between the decay factor and the risk at time 1: experiencing a "bad" risk at time 1 can create a greater concern for the potential experience at time 2 and therefore a larger decay factor.

This model can be seen as a two-stage backward procedure when assessing the global utility of a stream $\left(X_{1}, X_{2}\right)$. At time 1 , once the first risk $X_{1}$ has been experienced, the (conditional expected) utility of the remaining part $X_{2}$ is computed:

$$
U_{1}\left(X_{2} \mid X_{1}\right)=\beta\left(X_{1}\right) \mathbb{E}\left[u\left(X_{2}\right) \mid X_{1}\right],
$$

where $\beta\left(X_{1}\right)$ is a subjective weight. At the same time, the (contingent) utility of the first part is of course $u\left(X_{1}\right)$. Going backward, at time 0 the agent computes the expectation of the overall utility at time 1 , i.e.

$$
U\left(X_{1}, X_{2}\right)=\mathbb{E}\left[u\left(X_{1}\right)+U_{1}\left(X_{2} \mid X_{1}\right)\right]=\mathbb{E}\left[u\left(X_{1}\right)+\beta\left(X_{1}\right) u\left(X_{2}\right)\right] .
$$

Note that this state-dependent case is simple as we assume an explicit separation of the impact of the first risk experience on the assessment of the risk at the second time through this multiplicative factor $\beta$. The weight put by the agent on the risk he will experience at time 2 is all the more important so since his experience at time 1 has been damaging. Another possibility would be to let the coefficient characterizing the risk aversion of the agent depend on the previous risk experience. This would however require an explicit formulation of the utility function we consider.

The preference functionals in (20) may be written as $U\left(X_{1}, X_{2}\right)=\mathbb{E} w\left(X_{1}, X_{2}\right)$, where

$$
w\left(x_{1}, x_{2}\right)=u\left(x_{1}\right)+\beta\left(x_{1}\right) u\left(x_{2}\right) \text {. }
$$

We note that $w$ is not necessarily increasing in the first variable, but it is surely strictly increasing in the second one, as $\partial w / \partial x_{2}=\beta\left(x_{1}\right) u^{\prime}\left(x_{2}\right)>0$; therefore the functional $U$ satisfies the Non-Satiation Property $(\mathcal{N} S)$. Regarding the concavity of $w$ (and consequently that of $U$ ), the following Lemma provides the necessary framework ensuring that this property holds true, and therefore that the previous results on Pareto optimality can be applied:

Lemma 5.3 If $u$ and $\beta$ are twice differentiable and satisfy:

$$
\left(u^{\prime \prime}\left(x_{1}\right)+\beta^{\prime \prime}\left(x_{1}\right) u\left(x_{2}\right)\right) \beta\left(x_{1}\right) u^{\prime \prime}\left(x_{2}\right)-2\left(\beta^{\prime}\left(x_{1}\right) u^{\prime}\left(x_{2}\right)\right)^{2} \geq 0, \quad \forall\left(x_{1}, x_{2}\right) \in \mathbb{R}^{2}
$$

then the map $w$ defined in (21) is concave. 
Proof. If $u$ and $\beta$ are twice differentiable, then it is easy to prove that the map $w$ is twice differentiable. Computing its Hessian matrix $H_{w}$, it easily turns out that condition (22) is necessary and sufficient for $H_{w}$ to be positive semi-definite, which implies the concavity for $w$.

In this state-dependent setting, under the above conditions, it is possible to obtain a characterization of Pareto optimal allocations. However, the first order conditions become quite involved as we can see in the following proposition:

Proposition 5.2 Under the above specifications for $U_{A}$ and $U_{B}$ and assuming that condition (22) holds true, a (bounded) allocation $\left(\mathbf{X}^{A}, \mathbf{X}^{B}\right)$ is Pareto optimal if and only if there exists some constant $\lambda>0$ such that:

$\left\{\begin{array}{l}u_{A}^{\prime}\left(X_{1}^{A}\right)+\beta_{A}^{\prime}\left(X_{1}^{A}\right) \mathbb{E}\left[u_{A}\left(X_{2}^{A}\right) \mid \mathcal{F}_{1}\right]=\lambda\left(u_{B}^{\prime}\left(X_{1}^{B}\right)+\beta_{B}^{\prime}\left(X_{1}^{B}\right) \mathbb{E}\left[u_{B}\left(X_{2}^{B}\right) \mid \mathcal{F}_{1}\right]\right) \\ \beta_{A}\left(X_{1}^{A}\right) u_{A}^{\prime}\left(X_{2}^{A}\right)=\lambda \beta_{B}\left(X_{1}^{B}\right) u_{B}^{\prime}\left(X_{2}^{B}\right) .\end{array}\right.$

Proof. Since the functionals $U_{A}$ and $U_{B}$ satisfy the Non-Satiation Property $(\mathcal{N} S)$ and are concave (thanks to condition (22)), Theorem 4.5 remains valid. Computing the partial derivatives of $w$, it can be easily verified that the two equations (6) in this case become (23).

\subsection{An example with a non time-additive utility functional}

We now consider an example of a preference functional which is not timeadditive. Let $u$ be a utility function and $\beta>0$ a subjective decay factor and consider the following preference functional:

$$
U(\mathbf{X})=\mathbb{E} u\left(\sum_{n=1}^{N} \beta^{n-1} X_{n}\right)=\mathbb{E} u\left(X_{1}+\beta X_{2}+\ldots+\beta^{N-1} X_{N}\right) .
$$

In this case $U(\mathbf{X})=\mathbb{E} w(\mathbf{X})$, where

$$
w\left(x_{1}, \ldots, x_{N}\right)=u\left(\sum_{n=1}^{N} \beta^{n-1} x_{n}\right)
$$

is a standard utility function for processes. This map is clearly concave and strictly increasing in each variable.

Contrary to the previous specifications, the agent first cumulates the subjectively discounted risks and then computes the expected utility of the result. As a consequence, the subjective decay factors $\beta$ appearing in the two models have different meanings and cannot be directly compared. 
Focusing on a 2-period model, the two agents $A$ and $B$ are now characterized by the following preference functionals:

$$
U_{i}\left(X_{1}, X_{2}\right)=\mathbb{E} u_{i}\left(X_{1}+\beta_{i} X_{2}\right), i=A, B .
$$

for two utilities $u_{A}$ and $u_{B}$ and two fixed decay factors $\beta_{A}, \beta_{B}>0$. The corresponding utility functions for processes are:

$$
w_{i}\left(x_{1}, x_{2}\right)=u_{i}\left(x_{1}+\beta_{i} x_{2}\right), i=A, B .
$$

The preference structure defined by this type of functional is a priori not timeconsistent in the sense of Kreps and Porteus (1978) or Johnsen and Donaldson (1986). Moreover, this is not really a proper multi-period example as it could be seen as a simple one-period equivalent: the agents are indeed simply interested in their aggregated risks. They do as if there was a single cash-flow at time $t_{2}$. Therefore, it is not surprising to find the following results in terms of optimal allocations. The condition on the decay factor is however not so obvious.

Proposition 5.3 Under the above specification for $U_{A}$ and $U_{B}$,

1. If $\beta_{A} \neq \beta_{B}$ then there is no Pareto optimal allocation;

2. If $\beta_{A}=\beta_{B} \triangleq \beta$, the following statements are equivalent:

(a) The allocation $\left(X_{1}^{A}, X_{2}^{A}\right)$ is Pareto optimal;

(b) $X_{1}^{A}+\beta X_{2}^{A}=\psi\left(\widehat{X}_{1}+\beta \widehat{X}_{2}\right)$ where the map $\psi$ satisfies for all $x \in \mathbb{R}$, for some constant $\lambda>0$

$$
u_{A}^{\prime}(\psi(x))=\lambda u_{B}^{\prime}(x-\psi(x))
$$

(c) $X_{1}^{A}+\beta X_{2}^{A}=\psi\left(\widehat{X}_{1}+\beta \widehat{X}_{2}\right)$ where the map $\psi$ is differentiable and solves for any $x \in \mathbb{R}$

$$
\psi^{\prime}(x)=\frac{R A_{B}(x-\psi(x))}{R A_{A}(\psi(x))+R A_{B}(x-\psi(x))},
$$

where $R A_{i}$ denotes the Arrow-Pratt coefficient of absolute risk aversion agent $i(i=A, B)$.

Proof. The proof of this result is very similar to that of Proposition 5.1. Therefore we omit it. Note simply that in this case the appropriate choice of the constant $\lambda$ is $\lambda=\frac{u_{A}^{\prime}(\psi(0))}{u_{B}^{\prime}(-\psi(0))}$.

Remark 5.4 In this framework, the variables that matter are the cumulated risks of each agent, capitalized up to the last maturity date. Therefore, it is quite natural that the Pareto optimality of an allocation $\left(\mathbf{X}^{A}, \mathbf{X}^{B}\right)$ is determined only by the cumulated capitalized variables $\left(X_{1}^{A}+\beta X_{2}^{A}, X_{1}^{B}+\beta X_{2}^{B}\right)$. 
Remark 5.5 The obtained result is similar to Wyler's one in the one-period case (Proposition 2.14), replacing the aggregated risk with the cumulation of the aggregated risks. It is however impossible to specify explicitly the allocation rule at time 1 and at time 2, only the aggregate rule $\psi$ can be characterized, which is not surprising given the considered preference criterion. Note also that, on the contrary to the previous situation with cumulated discounted expected utilities, even if the cumulated allocation rule $\psi$ does not depend on the original risks, the individual Pareto optimal rules (at each intermediate date) may depend on the original individual risks.

Remark 5.6 The fact that when the agents do not agree on their decay factors, no Pareto-optimal risk allocation is possible between them is rather interesting, and may appear at first counter-intuitive. However, in this case, the agents do not agree on the risk they have to share, as only the cumulated risk matters and they use different cumulation rules. This disagreement on the decay factor may be the consequence of differences in the agents' anticipations or beliefs but also of some information asymmetry. In this latter situation, one can also think of the standard Principal-Agent problem in the insurance and economic literature: moral hazard or adverse selection have to be taken into account when designing a contract, as the buyer could have an impact on the considered risk (for more details, among a wide literature, refer for instance to the two papers on the relation Principal-Agent by Rees (1985a and 1985b)).

\subsection{Examples with exponential utilities}

We assume now that the (classical) utility functions entering the previous specifications given in Equations (15) and (24) are of exponential type, i.e. $u(x ; \gamma)=-\exp (-\gamma x), x \in \mathbb{R}$, where $\gamma>0$ is the risk aversion coefficient.

\subsubsection{State-independent time additive case}

The agents are characterized by the preference functionals:

$$
U_{i}\left(X_{1}, X_{2}\right)=\mathbb{E} u\left(X_{1} ; \gamma_{i}\right)+\beta_{i} \mathbb{E} u\left(X_{2} ; \gamma_{i}\right), i=A, B,
$$

where $\beta_{A}, \beta_{B}>0$ are the subjective decay factors and $\gamma_{A}, \gamma_{B}>0$ are the risk aversion coefficients.

Proposition 5.4 Under the above specification an allocation $\left(\mathbf{X}^{A}, \mathbf{X}^{B}\right)$ is Pareto optimal if and only if:

$$
X_{1}^{A}=\frac{\gamma_{B}}{\gamma_{A}+\gamma_{B}} \widehat{X}_{1}+c
$$




$$
X_{2}^{A}=\frac{\gamma_{B}}{\gamma_{A}+\gamma_{B}} \widehat{X}_{2}+\frac{1}{\gamma_{A}+\gamma_{B}} \log \frac{\beta_{A}}{\beta_{B}}+c,
$$

for some constant $c \in \mathbb{R}$.

Proof. In this case, the necessary and sufficient conditions in Part 1 of Proposition 5.1 for Pareto optimality become:

$$
\begin{gathered}
\gamma_{A} \exp \left(-\gamma_{A} X_{1}^{A}\right)=\lambda \gamma_{B} \exp \left(-\gamma_{B} X_{1}^{B}\right) \\
\beta_{A} \gamma_{A} \exp \left(-\gamma_{A} X_{2}^{A}\right)=\lambda \beta_{B} \gamma_{B} \exp \left(-\gamma_{B} X_{2}^{B}\right),
\end{gathered}
$$

for some $\lambda>0$. If we set

$$
c=\frac{1}{\gamma_{A}+\gamma_{B}} \log \left(\lambda \frac{\gamma_{B}}{\gamma_{A}}\right)
$$

then an easy computation shows that (29) (resp. (30)) is equivalent to (27) (resp. to (28)) under the identity $\widehat{X}_{1}=X_{1}^{A}+X_{1}^{B}$ (resp. $\widehat{X}_{2}=X_{2}^{A}+X_{2}^{B}$ ). Finally, note that (31) provides a one-to-one correspondence between $\lambda$ in $(0,+\infty)$ and $c$ in $\mathbb{R}$.

Remark 5.7 In this particular situation, the risk sharing at both dates is proportional and the coefficient of proportionality $\frac{\gamma_{B}}{\gamma_{A}+\gamma_{B}}$ is the same at both times: Agent A will bear the same ratio of the aggregated risk at each individual time. The agents' respective risk aversion does not evolve over time and the decay factor does not play any role in the risk sharing rule.

However, the decay factor does have a specific role through the correction term, i.e. the constant that also appears in the allocation rule. This correction term, which is not random, can be compared with an exchange of cash occurring at the same time as the exchange of risk, and therefore a natural interpretation is that of forward price, or cash adjustment (for swaps for instance).

At time 2, the correction term is composed of two parts, the first part is the same as for time 1, but there is an additional term, which depends on the decay factor and acts as a modification of this "cash adjustment". Therefore, as far as the "price" or "risk assessment" is concerned, the decay factor plays a similar role to the risk aversion coefficient for time 2 , and acts as a modifier of how risk averse the agent becomes between time 1 and time 2 . The greater (in relative value) the decay factor is, the more risk averse the agent becomes, and the less the agent wants to "pay" for this risk exchange. This is in line with the economic literature, where the discount factor, or more generally the decay factor, is seen as a parameter translating the preference for the present, and therefore the aversion for the future. 
Remark 5.8 Proposition 5.4 can be easily extended to the general case of $N \geq 2$ times. A Pareto optimal allocation can simply be written as:

$$
X_{n}^{A}=\frac{\gamma_{B}}{\gamma_{A}+\gamma_{B}} \widehat{X}_{n}+(n-1) b+c, \quad n \leq N
$$

where $c$ is an arbitrary constant and $b=\frac{1}{\gamma_{A}+\gamma_{B}} \log \frac{\beta_{A}}{\beta_{B}}$.

\subsubsection{Non time additive case}

The agents are characterized by the preference functionals:

$$
U_{i}\left(X_{1}, X_{2}\right)=\mathbb{E} u\left(X_{1}+\beta_{i} X_{2} ; \gamma_{i}\right), i=A, B,
$$

where $\beta_{A}, \beta_{B}>0$ are the subjective decay factors and $\gamma_{A}, \gamma_{B}>0$ are the risk aversion coefficients. We know from Proposition 5.3 that if $\beta_{A} \neq \beta_{B}$, then there does not exist any Pareto optimal allocation: we therefore assume $\beta_{A}=\beta_{B} \triangleq \beta$

Proposition 5.5 Under the above specification, an allocation is Pareto optimal if and only if:

$$
X_{1}^{A}+\beta X_{2}^{A}=\frac{\gamma_{B}}{\gamma_{A}+\gamma_{B}}\left(\widehat{X}_{1}+\beta \widehat{X}_{2}\right)-c,
$$

for some constant $c \in \mathbb{R}$.

Proof. Once we set $c=\frac{1}{\gamma_{A}+\gamma_{B}} \log \left(\frac{\gamma_{B}}{\gamma_{A}}\right)$, the proof is a consequence of Proposition 5.3 and the identities $\widehat{X}_{1}=X_{1}^{A}+X_{1}^{B}$ and $\widehat{X}_{2}=X_{2}^{A}+X_{2}^{B}$. Note that there is a one-to-one correspondence between $\lambda$ in $(0,+\infty)$ and $c$ in $\mathbb{R}$.

The allocation rule is quite interesting: the risk sharing of the aggregated risks of time 1 and time 2 is proportional, which is not surprising since we are assuming an exponential utility function.

Remark 5.9 A simple consequence of the above proposition is the fact that an allocation is Pareto optimal if and only if:

$$
X_{2}^{A}=\frac{\gamma_{B}}{\gamma_{A}+\gamma_{B}} \widehat{X}_{2}+\frac{1}{\beta}\left(\frac{\gamma_{B}}{\beta\left(\gamma_{A}+\gamma_{B}\right)} \widehat{X}_{1}-X_{1}^{A}\right)-c,
$$

for some constant $c \in \mathbb{R}$.

Therefore, the allocation of risk at time 1 can be completely arbitrary while the allocation at time 2 simply has to satisfy the above condition. The risk allocation at time 2 is of proportional type, coming in the form $X_{2}^{A}=$ $\frac{\gamma_{B}}{\gamma_{A}+\gamma_{B}} \widehat{X}_{2}-\tilde{c}$, as in the previous case (with the same coefficient of proportionality). However, in the present case the correction term $\tilde{c}$ is no longer a constant but 
does depend on the aggregated risk and the allocation at time 1 . The decay factor plays an important role here since it reduces the inter-temporal impact. The higher the decay factor, the "more unrelated" the two risk allocations of time 1 and time 2 are.

Remark 5.10 The above proposition can be easily extended to the general case of $N \geq 2$ times. A Pareto optimal allocation can simply be written as:

$$
\sum_{n=1}^{N} \beta^{n-1} X_{n}^{A}=\frac{\gamma_{B}}{\gamma_{A}+\gamma_{B}}\left(\sum_{n=1}^{N} \beta^{n-1} \widehat{X}_{n}\right)-c, \quad n \leq N
$$

where $c$ is an arbitrary constant.

Again, the aggregated Pareto optimal allocation does not depend on the initial risk allocations. However, each Pareto optimal allocation for each time $n$, $X_{n}^{A}$, can depend on the original initial risks.

\section{Concluding COMments}

In this paper, we have considered the problem of Pareto optimal allocations in a general framework, when each of the two agents involved in the transaction is characterized by a general preference functional defined on a real vector space. The optimization problem is equivalent to a modified sup-convolution problem of the agents preference functionals. An immediate application of these results is the multi-period setting, involving risk streams taking place at different dates and potentially leading to risk exchanges at different dates. Some further characterization of Pareto optimality is then obtained for the wide class of expected utilities for processes. A potential applications of these results is the design of contracts depending on some long-term risks such as longevity risk.

The characterization of Pareto optimal allocations obtained on a real vector space can also be applied to the design of hybrid transactions based upon different types of risks. This may have some interesting implications in credit risk securitization for instance, or in bespoke transactions (such as the cession of some parts of a bank's portfolio to a hedge fund), for which the underlying risk has several components, including systematic and specific components.

Some further extensions of these results seem to be rather natural: in particular, studying the situation, where more than two agents are involved, can help to better understand the mechanisms behind some illiquid markets. This does not appear to be fundamentally different and one may expect the same type of results to hold true. The study of other economically relevant examples would also be extremely interesting: for instance the case of recursive utilities, as in Epstein and Zin (1989), or habit formation as in Dunn and Singleton (1986) or Ryder and Heal (1973). 
Finally, an interesting question could be the inference of the preference functionals of the different agents from existing transactions, in the spirit of the studies of Chiappori, Ekeland, Kubler and Polemarchakis (2002) or Dybvig and Rogers (1997). Given some past transactions (OTC contracts or reinsurance treaties for instance), what can we deduct from the preferences of the counterpart and how can this impact further transactions with this particular agent? This question is essential when considering the design of future deals as to ensure the feasibility of these transactions, and this will be the topic for further research.

\section{REFERENCES}

Aase, K. (2002) Perspectives of risk sharing. Scandinavian Actuarial Journal 2, 73-128.

ACCIAIO, B. (2007) Optimal risk sharing with non-monotone monetary functionals. Finance and Stochastics 11, 267-289.

Aliprantis, C.D. and Border, K.C. (1999) Infinite dimensional analysis. 2nd Ed. Springer Verlag.

Barrieu, P. and El Karoui, N. (2005) Inf-convolution of risk measures and optimal risk transfer. Finance and Stochastics 9, 269-298.

Barrieu, P. and El Karoui, N. (2006) Pricing, hedging and optimally designing derivatives via minimization of risk measures. In Volume on Indifference Pricing (ed: R. Carmona), Princeton University Press (to appear), Preprint Ecole Polytechnique nb 581 (http://www.cmap. polytechnique.fr/preprint/repository/581.pdf).

Borch, K. (1960) The safety loading of reinsurance premiums. Skandinavisk Aktuarietidsskrift 43, $163-184$.

BorCH, K. (1960) Reciprocal reinsurance treaties seen as a two-person cooperative game. Skandinavisk Aktuarietidsskrift, 29-58.

BorCH, K. (1960) Reciprocal reinsurance treaties. ASTIN Bulletin 1, 170-191.

BorCH, K. (1962) Equilibrium in a reinsurance market. Econometrica 30, 424-444.

BüHLmann, H. (1970) Mathematical methods in risk theory. Springer Verlag.

BüHLManN, H. (1980) An economic premium principle. ASTIN Bulletin 11, 52-60.

BüHLManN, H. (1984) The general economic premium principle. ASTIN Bulletin 14, 13-21.

Bühlmann, H. and Jewell, W.S. (1979) Optimal risk exchanges. ASTIN Bulletin 10, 243-262.

Chitappori, P., Ekeland, I., Kubler, F. and Polemarchakis, H. (2002) The identification of preferences from equilibrium prices: uncertainty. Journal of Economic Theory 102, 403-420.

Dana, R.A. and JeAnblanc, M. (2002) Financial markets in continuous time, valuation and equilibrium. Springer Verlag.

Duffie, D. (1996) Dynamic Asset Pricing Theory. Princeton University Press (second edition).

Du Mouchel, W. (1968) The Pareto optimality of an $n$-company reinsurance treaty. Skandinavisk Aktuarietidsskrift 51, 165-170.

DunN, K. and Singleton, K. (1986) Modeling the term structure of interest rates under nonseparable utility and durability of goods. Journal of Financial Economics 17, 27-55.

DybbVig, P. and Rogers, C. (1997) Recovery of preferences from observed wealth in a single realization (1997). Review of Financial Studies 10, 151-174.

El Karoui, N. and Ravanelli, C. (2007) Cash sub-additive risk measures and interest rate ambiguity. Preprint Ecole Polytechnique nb 608 (http://www.cmap.polytechnique.fr/preprint/ repository/608.pdf).

EPSTEIN, L. and ZIN, S. (1989) Subsitution, risk aversion and the temporal behavior of consumption and asset returns I: A theoretical framework. Econometrica 57, 937-969.

Föllmer, H. and Schied, A. (2004) Stochastic finance: An introduction in discrete time. De Gruyter Studies in Mathematics (revised edition).

Gerber, H.U. (1978) Pareto optimal risk exchanges and related decision problems. ASTIN Bulletin 10, 25-33. 
Golubin, A.Y. (2005) Pareto-optimal Contract in an Insurance Market. ASTIN Bulletin 35, 363378.

Johnsen, T. and Donaldson, J. (1986) The structure of intertemporal preferences under uncertainty and time consistent plans. Econometrica 53, 1451-1458.

Jouini, E., SCHACHERMAYER, W. and TouZI, N. (2008) Optimal risk sharing for law invariant monetary utility functions. Mathematical Finance 18(2), 269-292.

Kreps, D. and Porteus, E. (1978) Temporal resolution of uncertainty and dynamic choice. Econometrica 46, 185-200.

Mas-Colell, A. (1985) The theory of general economic equilibrium - a differentiable approach. Cambridge University Press.

Mas-Colell, A. (1986) Valuation equilibrium and Pareto optimum revisited. Chapter 17 in: Contributions to Mathematical Economics, Hon. G. Debreu, 317-331.

Raviv, A. (1979) The design of an optimal insurance policy. American Economic Review 69, 8496.

ReEs, R. (1985a) The Theory of Principal and Agent, part I. Bulletin of Economic Research 37, 3-26.

ReEs, R. (1985b) The Theory of Principal and Agent, part II. Bulletin of Economic Research 37, 75-95.

Ryder, H. and Heal, G. (1973) Optimal growth with intertemporally dependent preferences. Review of Economic Studies 40, 1-31.

Savina, O. (2007) Optimal design of Cat-bonds involving utilities. Part III of draft PhD thesis, Department of Statistics, London School of Economics.

WiLSON, R. (1968) The theory of syndicates. Econometrica 36, 119-131.

WYLER, E. (1990) Pareto-optimal risk exchanges and a system of differential equations: a duality theorem. ASTIN Bulletin 20, 23-32.

Pauline Barrieu

Department of Statistics

London School of Economics

Houghton Street, WC2A 2 AE London

United Kingdom

E-mail:p.m.barrieu@lse.ac.uk

Giacomo ScANDOLO

Department of Mathematics for Economics

University of Florence

via Lombroso 6/17, 50134 Florence

Italy

E-mail: giacomo.scandolo@unifi.it 